Pteridines

Vol. 15, 2004, pp. $107-112$

\title{
Neopterin: a Mediator of the Cellular Immune System*
}

\author{
Georg Hoffmann, Wolfgang Schobersberger \\ Institute for Leisure, Travel, and Alpine Medicine: University for Health Sciences, \\ Medical Informatics and Technology, Austria \\ *Dedicated to Prof. Dr. Dr. h. c. Helmut Wachter on the occasion of his $75^{\text {th }}$ birthday
}

\begin{abstract}
In this review, we will summarize our investigations on the potential biochemical, physiological, and pathophysiological effects of neopterin in the course of infection and inflammation. In a number of in vitro-studies we could show that the pteridine compound neopterin manifested distinct biochemical properties via interfering with cellular redox mechanisms. Among the observed actions of neopterin are the stimulation of inducible nitric oxide synthase gene expression and nitric oxide release, the activation of the transcription factor nuclear factor- $\kappa \mathrm{B}$, the induction of apoptotic ccll death, the inhibition of erythropoietin synthesis and release, and the stimulation of tumor necrosis factor- $\alpha$ generation in different cell culture as well as organic models. By these means, neopterin may represent a part of the host-defence reactions. In addition, an excessive or continous release of neopterin due to a chronically activated cellular immune system may contribute to the deleterious events commonly observed in progressive infectious or inflammatory diseases. Our data are supported by the findings of a strong correlation between raised levels of neopterin and the severity and the outcome of these disorders.
\end{abstract}

Key words: neopterin, infection, inflammation, oxidative stress

\section{Introduction}

Since its isolation in 1967 by Sakurai and Goto (1), neopterin has been shown to be a useful indicator for an activated cellular immune system in humans. Studies by Wachter and co-workers reported increased levels of neoptcrin in serum, urine, synovial and cerebrospinal fluid or saliva in diseases characterized by a stimulation of the cell-mediated immune response. Thus, high neopterin concentrations are a reliablc indicator of the host-defence reaction in infectious or malignant diseases. Furthermore, neopterin levels are augmented during graft rejection with or without infectious complications. Due to its quality as a disease marker, neopterin measurements have become a significant tool for blood and organ donor screenings (2-6).

Our specific interest in neopterin focused on its potential biochemical and physiological (or pathophysiological) effects in the course of infectious diseases. Early studies suggested the promotion of oxidative stress to be a key principle of neopterin's mode of action. In cell-free solutions, neopterin enhanced chlo- ramine $T$ - and hydrogen peroxide-mediated chemiluminescence. Since these effects depended on the presence of transition metals, it was hypothesized that neopterin stimulates the production of hydroxyl radicals (7-9). In our own studies, we investigated the potential effects of neopterin in various cell culturc systems and organic models. The results with respect to their clinical implications will be summarized in this review.

\section{Biochemical effects of neopterin}

Reactive nitrogen species derived from nitric oxide are produced in excessive amounts in a number of infectious diseases, e.g. sepsis and septic shock. Due to its vasodilatory effects, nitric oxide (NO) is discussed to be a paramount mediator of the detrimental hypotensive reactions in the course of endotoxemia. Raised levels of serum neopterin have been reported in sepsis and there was a correlation between the concentration of this pteridine compound and the severity as well as

Correspondence to: PD Dr. Georg Hoffmann, Institute for Leisure, Travel, and Alpine Medicine, University for Health Sciences, Medical Informatics and Technology, Innrain 98, 6020 Innsbruck, Austria Phone: ++43-50-8648-3856, Fax: ++43-50-8648-673840, c-mail: georg.hoffmann@umit.at 
the outcome of the disease (10). In our studies using vascular smooth muscle as a ccllular model, we obscrved a neopterin-initiated expression of inducible nitric oxide synthase (iNOS) (Fig. 1) and a subsequent

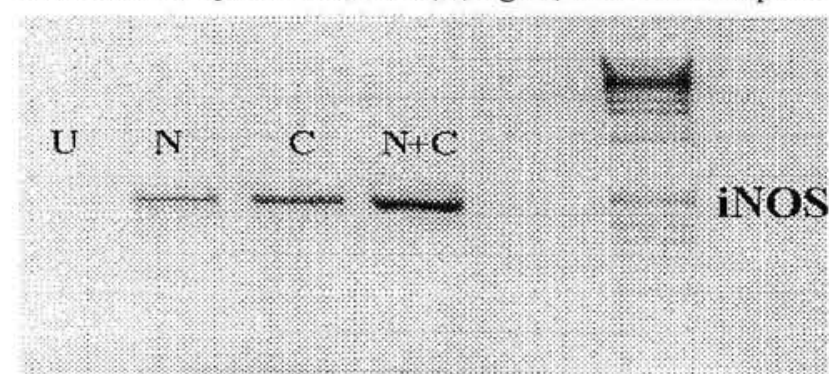

Figure 1. Qualitative analyses of inducible nitric oxide synthase (iNOS) gene expression in vascular smooth muscle cells following a 6-h incubation with neopterin $(10 \mu \mathrm{M})$, a combination of tumor necrosis factor- $\alpha(500 \mathrm{U} / \mathrm{ml})$ and interferon- $\gamma(100 \mathrm{U} / \mathrm{ml})(\mathrm{C})$, and a mixture of neopterin plus cytokines $(\mathrm{N}+\mathrm{C}) . \mathrm{U}$ represents unstimulated controls.

increase of NO generation which was most likely triggered via an activation of the transcription factor nuclear factor- $\kappa \mathrm{B}(\mathrm{NF}-\kappa \mathrm{B})$ by neopterin. NF- $\kappa \mathrm{B}$ activation may provide a link between neopterin, oxidative stress, and iNOS gene expression. Morcover, neopterin-induced NO synthesis may explain the observations made in endotoxemic animals and humans, were elevations of neopterin concentrations were accompanied by an increase in nitrite/nitrate levels $(11,12)$. Pro-inflammatory cytokines, especially tumor necrosis factor- $\alpha$ (TNF- $\alpha$ ) have been reported to be involved in the pathogenesis of sepsis and septic shock as well $(13,14)$. Considering synthesis and secretion, similarities between neopterin and TNF- $\alpha$ were shown. For example, neopterin was found to amplify the secretion of TNF- $\alpha$ from activated peripheral blood mononuclear cells (15). We could identify neopterin to be a potent stimulus of TNF- $\alpha$ gene expression (Fig. 2) as well as TNF- $\alpha$ protein synthesis

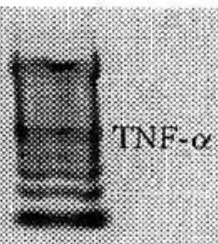

Figure 2. Qualitative analyses of tumor necrosis factor- $\alpha$ (TNF- $\alpha$ ) gene expression in vascular smooth muscle cells following a 3 -h incubation with ncopterin at increasing concentrations: $1 \mu \mathrm{M}(\mathrm{N} 1), 10 \mu \mathrm{M}$ (N10), $100 \mu \mathrm{M}(\mathrm{N} 100)$, and $1000 \mu \mathrm{M}$ (N1000). U represents unstimulated controls. in vascular smooth muscle cells (16). Via the affection of septic mediators like NO or TNF- $\alpha$, the synthesis of neopterin may contribute to the deleterious events in the course of bacterial infections (Fig. 3). This hypothesis is further supported by the observation of disturbed coronary flow and cardiac contractility following neopterin perfusion in the isolated rat heart (17).

In addition to raising NO levels, neopterin was found to induce apoptotic death in vascular smooth muscle cells (18). With respect to intracellular signal transduction mechanisms, there might be a connection between neopterin-induced nitric oxide generation and neopterin-mediated programmed cell death. In fact, the simultaneous incubation of smooth muscle cells with neopterin plus an inhibitor of the iNOS enzyme resulted in a loss of neopterin's impact on apoptosis in our studies (18). In blood vesscls, the combination of raised levels of neopterin, apoptotic cell death, and nitric oxide generation is found in the process of atherusclerosis (19-21). Although the exact role of apoptosis in atherogenesis is not clear to date, stimulation of iNOS gene expression by neopterin might represent an important step towards programmed cell death under these conditions. In the early phase of atherosclerosis, this might trigger a beneficial limitation of plaque growth while in the later steps, apoptosis may contribute to a destabilization of massive plaques and may thus promote a pro-thrombotic state.

The concept of neopterin acting as a pro-apoptotic mediator could be confirmed in studies using the alveolar epithelial type II-like cell line L2. Single application of neopterin increased the rate of apoptosis in these cells. Furthermore, additive effects of neopterin and the pro-inflammatory cytokines TNF- $\alpha$ and interferon- $\gamma$ (IFN- $\gamma$ ) could be observed (Fig. 4). In contrast to vascular smooth muscle cells, neopterin-induced programmed L2-cell death was not affected by iNOSinhibition or the application of NO-donors. This suggests that apoptosis in L2 cells triggerd by neopterin does not involve the production of NO (22). Within the pulmonary system, apoptosis is supposed to be a significant event in the pathogenesis and the course of inflammatory diseases of the lung, e.g. sarcoidosis, tuberculosis, and lung cancer (23). A combined action of neopterin and other pro-inflammatory mediators like TNF- $\alpha$ might contribute to the deterioration of pulmonary functions under these conditions. This hypothesis is further supported by the observations of an increased expression and release of intercellular adhesion molecule-1 (ICAM-1) in L2 cells following incubation with neopterin (24). ICAM-1 mediates the migration and activation of leukocytes. The accumulation of macrophages, neutrophils, or lymphocytes as well as their secretory products at the site of infection 
Inititating event:

- endotoxins

- exotoxins

- enterotoxins

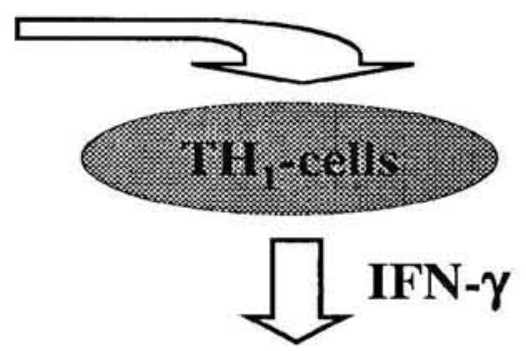

Neopterin, TNF- $\alpha$<smiles>C1CCCC1</smiles>

NF- $\kappa \mathrm{B}$ activation, iNOS gene expression, TNF- $\alpha$ synthesis

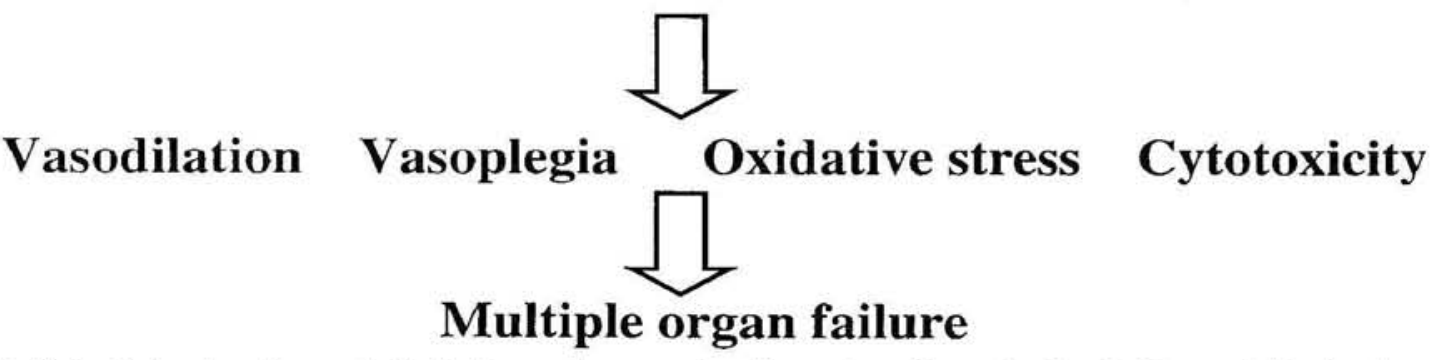

Figure 3. Potential role of neopterin in the pathogenesis of sepsis and septic shock. Bacterial infections lead to the activation of TH1-type lymphocytes. The ensuing release of interferon- $\gamma$ (IFN- $\gamma$ ) activatcs monocytes/macrophages to produce and release neopterin. As a single stimulus or in addition to cytokines like tumor necrosis factor- $\alpha$ (TNF- $\alpha$ ), neopterin triggers pro-inflammatory events. Among those, inducible nitric oxide synthase (iNOS) gene expression and the subsequent release of nitric oxide in excessive amounts favours the pathophysiological hypotensive reaction characteristic for septic shock.

is part of the unspecific host-defence reaction. However, a continous stimulation of these events may result in damage of the airway epithelium. Although neopterin and ICAM-1 synthesis are not generally connected in infectious diseases, a significant correlation between both compounds could be described in the broncho-alveolar lavage fluid in patients with sarcoidosis (25). Taken together, these data as well as our own in vitro-experiments point towards a role of neopterin in the regulation of ICAM-1 synthesis within the alvolar epithelium.

Chronically increased activities of the cellular immune system like sepsis and others described in this review are regularly associated with anaemia $(26,27)$. A major trigger for anaemia under these conditions is the suppression of erythropoietin (EPO) production by pro-inflammatory mediators, e.g. TNF- $\alpha$, interleukin$1 \beta$, (IL-1 $\beta$ ), or bacterial lipopolysaccharides (28-30). Various data provided evidence that neopterin might be involved in the onset of anaemia, too. Thus, the highest concentrations of neopterin in cancer patients are reported in tumors associated with the most pronounced degree of anaemia (31), and inverse correlations have been observed between scrum concentrations of neopterin and iron as well as transferrin (32). Indeed, neopterin proved to suppress hypoxia-induced EPO generation in the human hepatoma cell line $\mathrm{HepG}_{2}$ (33). These observations could be confirmed in a subsequent study using isolated, hypoxically perfused rat kidney. Addition of neopterin into the perfusate strongly reduced hypoxia-stimulated EPO release (34). So far, no involvement of oxidative stress in these processes could be established, since the antioxidative compound $\mathrm{N}$-acetylcysteine did not change the effects of ncoptcrin on hypoxia-triggered EPO synthesis in our organ model (35). In addition, changes in intracellular calcium concentrations $\left[\mathrm{Ca}^{2+}\right] \mathrm{i}$ cannot serve as an explanation for the observed influence of neopterin on EPO generation, since verapamil did not affect the suppressed EPO-release following neopterin 

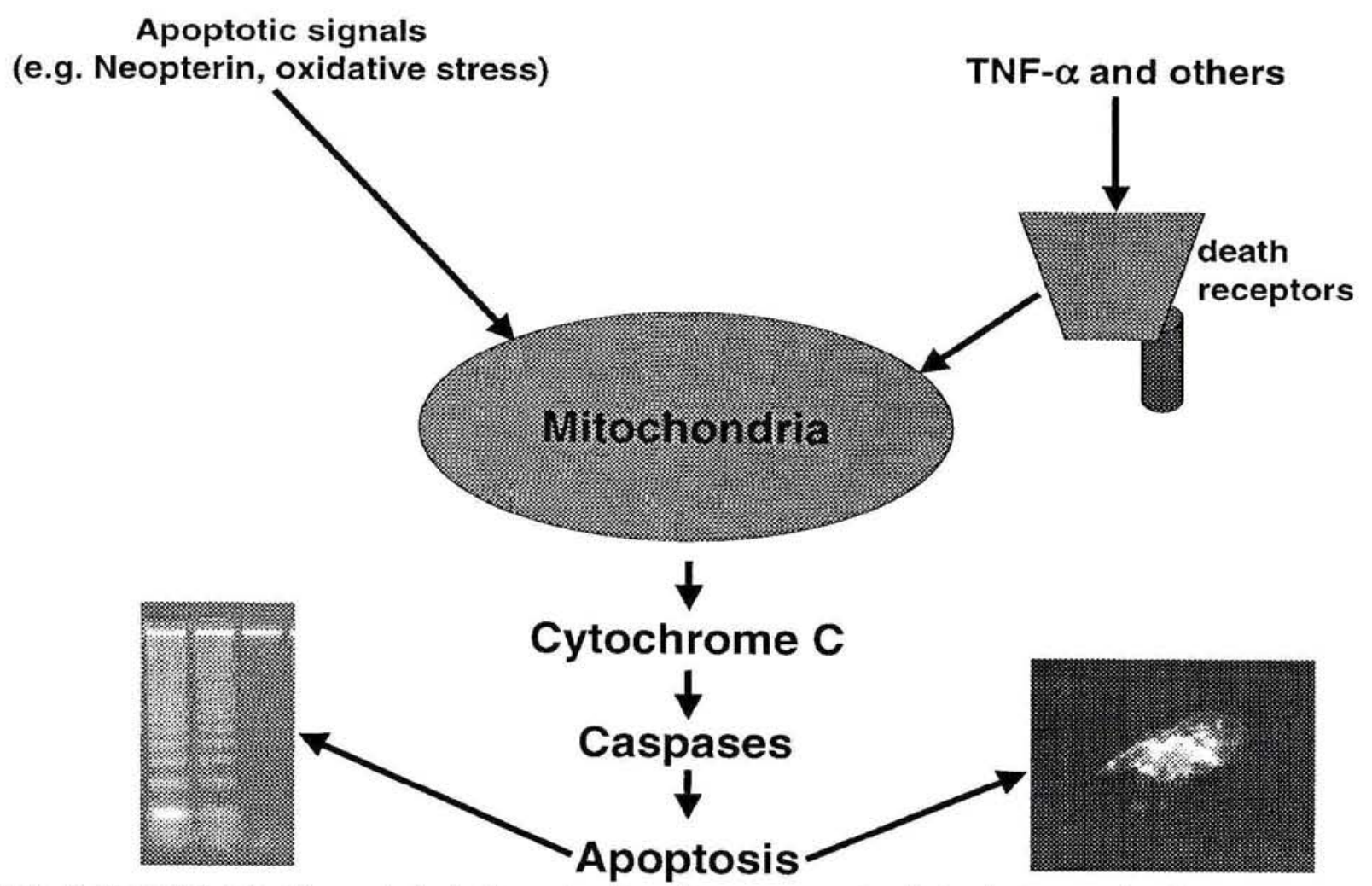

Figure 4. Potential role of neopterin in the activation of programmed cell death (apoptosis). Pro-apoptotic signals trigger the release of cytochrome $C$ from mitochondria. Subsequently, a cascade of enzymes (caspases) is activated which finally leads to the characteristic events of apoptosis, e.g. DNA fragmentation (which can be visualized via agarose gel electrophoresis of isolated cellular DNA, left insert) or blebbing of cells (which can be visualized via fluorescence microscopy, right insert). Most likely, neopterin acts via oxidative stress pathways to initiate apotosis, while cytokines like tumor necrosis factor- $\alpha$ (TNF- $\alpha$ ) require specific cell death receptors and different intracellular signalling mechanisms to activate mitochondria. This allows for additive or synergistic effects of both compounds with respect to activation of programmed cell death.

treatment.

However, this last observation does not exclude a potential interaction between neopterin and shifts in intracellular calcium. In a human myelomonocytic cell line, neopterin was found to increase $\left[\mathrm{Ca}^{2+}\right] \mathrm{i}(36)$. With respect to monocytic cells, elevation of $\left[\mathrm{Ca}^{2+}\right] \mathrm{i}$ contributes to the activation of NADPH oxidase and may thus promote the production of reactive oxygen species. In contrast to these findings, we found that neopterin inhibited $\mathrm{Ca}^{2+}$ transients triggered by ATP in L2 cells, suggesting that neopterin interferes with $\left[\mathrm{Ca}^{2+}\right] \mathrm{i}$ in a cell-type specific manner (37). Considering the importance of an increase in cytoplasmic $\mathrm{Ca}^{2+}$ for surfactant release by alveolar type II epithelial cells, disturbances of cellular calcium homeostasis by neopterin may contribute to the pulmonary dysfunctions in the course of inflammatory processes.

\section{Conclusions}

The above-mentioned findings provide evidence that neopterin acts as a mediator of host-defence reactions most likely via interfering with cellular redox systems. Although the exact role and the importance of neopterin in the course of infection and inflammation still remains unclear, the pteridine compound appears to be a part of the extracellular arsenal of IFN- $\gamma$-stimulated macrophages. Following an ineffective acute immune response, the chronic activation of neopterin synthesis and release may contribute to various adverse effects observed under these conditions. The findings summarized in this review may shed further insight into the complex mechanisms of a chronically activated cellular immune system and may provide an explanation for the commonly observed correlations between raised levels of neopterin and the severity of infectious diseases.

\section{Aknowledgements}

The present review summarizes a great deal of our scientific work over the past decade. The success we 
had in investigating the role of neopterin as a mediator of the cellular immune system would not have been possible without the constant support of others. The following list is incomplete at best. We would like to thank Ms. M. Schloesser, Ms. M. Czechowski, and Ms. M. Seibel for their excellent technical assistance as well as Prof. D. Fuchs and Prof. H. Wachter for their scientific input and encouragement.

\section{References}

1 Sakurai A, Goto M. Neopterin: isolation from human urine. J Biochem (Tokyo) 1967; 61: 142145.

2 Fuchs D, Granditsch G, Hausen A, Reibnegger G, Wachter $\mathrm{H}$. Urinary neopterin excretion in coeliac disease. Lancet 1983; 2: 463-464.

3 Fuchs D, Hausen A, Kofler M. Kosanowski H, Reibnegger G, Wachter H. Neopterin as an index of immune response in patients with tuberculosis. Lung 1984; 162: 337-346.

4 Fuchs D, Spira TJ, Hausen A, et al. Neopterin as a predictive marker for disease progression in human immunodeficiency virus type 1 infection. Clin Chem 1989; 35: 1746-1749.

5 Honlinger M, Fuchs D, Hausen A, et al. [Serum neopterin determination for the additional safeguarding of blood transfusions. Our experiences with 76,587 blood donors]. Dtsch Med Wochenschr 1989; 114: 172-176.

6 Wachter H, Hausen A, Grassmayr K. [Increased urinary excretion of neopterin in patients with malignant tumors and with virus diseases (author's transl)]. Hoppe Seylers Z Physiol Chem 1979; 360: 1957-1960.

7 Kojima S, Icho T, Mori H, Arai T. Enhancing potency of neopterin toward B-16 melanoma cell damage induced by UV-A irradiation and its possible application for skin tumor treatment. Anticancer Res 1995; 15: 1975-1980.

8 Murr C, Fuchs D, Gossler W, et al. Enhancement of hydrogen peroxide-induced luminol-dependent chemiluminescence by neopterin depends on the presence of iron chelator complexes. FEBS Lett 1994; 338: 223-226.

9 Weiss G, Fuchs D, Hausen A, et al. Neopterin modulates toxicity mediated by reactive oxygen and chloride species. FEBS Lett 1993; 321: 89-92.

10 Strohmaier W, Redl H, Schlag G, Inthorn D. D-erythro-neopterin plasma levels in intensive care patients with and without septic complications. Crit Care Med 1987; 15: 757-760.

I) Holfmann G, Schobersberger W, Frede S, et al.
Neopterin activates transcription factor nuclear factor-kappa B in vascular smooth muscle cells. FEBS Lett 1996; 391: 181-184.

12 Schobersberger W, Hoffmann G, Grote J, Wachter $\mathrm{H}$, Fuchs D. Induction of inducible nitric oxide synthase expression by neopterin in vascular smooth muscle cells. FEBS Lett 1995; 377: 461464.

13 Beutler B, Grau GE. Tumor necrosis factor in the pathogenesis of infectious diseases. Crit Care Med 1993; 21: S423-S435.

14 Offner F, Philippe J, Vogelaers D, et al. Serum tumor necrosis factor levels in patients with infectious disease and septic shock. J Lab Clin Med 1990; 116: 100-105.

15. Barak M, Gruener N. Neopterin augmentation of tumor necrosis factor production. Immunol Lett 1991; 30: 101-106.

16 Hoffmann G, Frede S, Kenn S, et al. Neopterininduced tumor necrosis factor-alpha synthesis in vascular smooth muscle cells in vitro. Int Arch Allergy Immunol 1998; 116: 240-245.

17 Margreiter J, Schlager A, Balogh A, et al. Exogenous neopterin causes cardiac contractile dysfunction in the isolated perfused rat heart. $\mathrm{J} \mathrm{Mol}$ Cell Cardiol 2000; 32: 1265-1274.

18 Hoffmann G, Kenn S, Wirleitner B, et al. Neopterin induces nitric oxide-dependent apoptosis in rat vascular smooth muscle cells. Immunobiology 1998; 199: 63-73.

19 Buttery LD, Springall DR, Chester AH, et al. Inducible nitric oxide synthase is present within human atherosclerotic lesions and promotes the formation and activity of peroxynitrite. Lab Invest 1996; 75: 77-85.

20 Herpfer I, Greilberger J, Ledinski G, Widner B, Fuchs D, Jurgens G. Neopterin and 7,8-dihydroneopterin interfere with low density lipoprotein oxidation mediated by peroxynitrite and/or copper. Free Radic Res 2002; 36: 509-520.

21 Weiss G, Willeit J, Kiechl S, et al. Increased concentrations of neopterin in carotid atherosclerosis. Atherosclerosis 1994; 106: 263-271.

22 Schobersberger W, Hoffmann G, Hobisch-Hagen P, et al. Neopterin and 7,8-dihydroneopterin induce apoptosis in the rat alveolar epithelial cell line L2. FEBS Lett 1996; 397: 263-268.

23 Compton CN, Franko AP, Murray MT, Diebel LN, Dulchavsky SA. Signaling of apoptotic lung injury by lipid hydroperoxides. J Trauma 1998; 44: 783788.

24 Hoffmann G, Rieder J, Smolny M, et al. Neopterininduced expression of intercellular adhesion molecule-1 (ICAM-1) in type II-like alveolar epithelial 
cells. Clin Exp Immunol 1999; 118: 435-440.

25 Baumer I, Zissel G, Schlaak M, Muller-Quernheim J. Shed soluble ICAM-1 molecules in bronchoalveolar lavage cell supernatants and serum of patients with pulmonary sarcoidosis. Lung 1997; 175: 105116.

26 Schobersberger W, Hobisch-Hagen P, Hoffmann G, Fuchs D. Erythropoietin response and cytokines in critically ill patients. Intensive Care Med 1997; 23: 920.

27 Schobersberger W, Hobisch-Hagen P, Fuchs D, Hoffmann G, Jelkmann W. Pathogenesis of anaemia in the critically ill patient. Crit Int Care 1998; 9: 111-117.

28 Faquin WC, Schneider TJ, Goldberg MA. Effect of inflammatory cytokines on hypoxia-induced erythropoictin production. Blood 1992; 79: 1987. 1994.

29 Jelkmann W, Pagel H, Wolff M, Fandrey J. Monokines inhibiting erythropoietin production in human hepatoma cultures and in isolated perfused rat kidneys. Life Sci 1992; 50: 301-308.

30 Udupa KB, Lipschitz DA. Endotoxin-induced suppression of crythropoiesis: the role of erythropoictin and a heme synthesis stimulating factor. Blood 1982; 59: 1267-1271.

31 Fuchs D, Zangerle R, Artner-Dworzak E, et al. Association between immune activation, changes of iron metabolism and anaemia in patients with HIV infection. Eur J Haematol 1993; 50: 90-94.
32 Denz H, Huber P, Landmann R, Orth B, Wachter H, Fuchs D. Association between the activation of macrophages, changes of iron metabolism and the degree of anaemia in patients with malignant disorders. Eur J Haematol 1992; 48: 244-248.

33 Schobersberger W, Jelkmann W, Fandrey F, Frede S, Wachter H, Fuchs D. Neopterin-induced suppression of erythropoietin production in vitro. Pteridines 1995; 8: 12-16.

34 Pagel H, Fandrey J, Schobersberger W, Fuchs D, Jelkmann W. Effects of neopterin and 7,8-dihydroneopterin on hypoxia-induced renal erythropoietin production. Eur J Haematol 1999; 62: 341-345.

35 Pagel H, Hoffmann G, Fuchs D, Schobersberger W. On the mechanisms of the neopterin-induced suppression of renal erythropoietin production. Pteridines 2004; 15: 28-32.

36 Woll E, Weiss G, Fuchs D, Lang F. Wachter H. Effect of pteridine derivatives on intracellular calcium concentration in human monocytic cells. FEBS Lett 1993; 318: 249-252.

37 Hoffmann G, Gollnick F, Meyer R. Neopterin inhibits ATP-induced calcium release in alveolar epithelial cells in vitro. Mediators Inflamm 2002; 11: 181-185. 